\title{
Does the structure of Pop III supernova ejecta affect the elemental abundance of extremely metal-poor stars?
}

\author{
Gen Chiaki ${ }^{1 \star}$ and Nozomu Tominaga ${ }^{2,3}$ \\ ${ }^{1}$ Center for Relativistic Astrophysics, School of Physics, Georgia Institute of Technology, Atlanta, GA 30332, USA \\ ${ }^{2}$ Department of Physics, Konan University, 8-9-1 Okamoto, Kobe, 658-0072, Japan \\ ${ }^{3}$ Kavli Institute for the Physics and Mathematics of the Universe (WPI), The University of Tokyo, \\ 5-1-5 Kashiwanoha, Kashiwa, Chiba 277-8583, Japan
}

\begin{abstract}
The first generation of metal-free (Pop III) stars are crucial for the production of heavy elements in the earliest phase of structure formation. Their mass scale can be derived from the elemental abundance pattern of extremely metal-poor (EMP) stars, which are assumed to inherit the abundances of uniformly mixed supernova (SN) ejecta. If the expanding ejecta maintains its initial stratified structure, the elemental abundance pattern of EMP stars might be different from that from uniform ejecta. In this work we perform numerical simulations of the metal enrichment from stratified ejecta for normal core-collapse $\mathrm{SNe}(\mathrm{CCSNe})$ with a progenitor mass $25 \mathrm{M}_{\odot}$ and explosion energies $0.7-10 \mathrm{~B}\left(1 \mathrm{~B}=10^{51} \mathrm{erg}\right)$. We find that $\mathrm{SN}$ shells fall back into the central minihalo in all models. In the recollapsing clouds, the abundance ratio $[\mathrm{M} / \mathrm{Fe}]$ for stratified ejecta is different from the one for uniform ejecta only within \pm 0.4 dex for any element M. We also find that, for the largest explosion energy (10 $\mathrm{B})$, a neighboring halo is also enriched. Only the outer layers containing $\mathrm{Ca}$ or lighter elements reach the halo, where $[\mathrm{C} / \mathrm{Fe}]=1.49$. This means that C-enhanced metalpoor (CEMP) stars can form from the CCSN even with an average abundance ratio $[\mathrm{C} / \mathrm{Fe}]=-0.65$.
\end{abstract}

Key words: galaxies: evolution — ISM: abundances — stars: formation — stars: low-mass — stars: Population III — stars: Population II

\section{INTRODUCTION}

The first generation of metal-free (Population III; Pop III) stars enrich the early Universe with the first heavy elements, affecting later structure formation. Pop III stars form in dark matter halos with masses $\sim 10^{5}-10^{6} \mathrm{M}_{\odot}($ minihalos; $\mathrm{MH})$ mainly at redshifts $\sim 10-30$. If they are massive $\left(\geq 8 \mathrm{M}_{\odot}\right)$, metals ejected from their supernova $(\mathrm{SNe})$ can enhance star formation (e.g., Ritter et al. 2016). The efficiency of their feedback depends on stellar mass and SN explosion energy, which in turn change the luminosity and metal ejecta mass, respectively. Theoretical works suggest that Pop III stars are massive $\left(M_{\text {PopIII }} \sim 10-1000 \mathrm{M}_{\odot}\right)$ because gas cooling caused by only molecular hydrogen is inefficient, and their parent clouds are stable against fragmentation (Bromm et al. 1999; Abel et al. 2002; Hirano et al. 2014; Susa et al. 2014). Although recent numerical studies have found that low-mass Pop III stars $\left(\lesssim 1 \mathrm{M}_{\odot}\right)$ can form through the fragmentation of accretion disks (e.g., Clark et al. 2011; Greif

^ E-mail: gen.chiaki@physics.gatech.edu et al. 2012; Stacy \& Bromm 2014), we restrict our focus on massive stars to study their metal enrichment.

A number of previous works have attempted to constrain the mass of Pop III stars. One approach is to directly observe Pop III stars, but no metal-free stars have so far been found albeit the large survey programs of stars in the Milky Way and neighboring dwarf galaxies, such as the HK (Beers et al. 1985, 1992), Hamburg/ESO (Christlieb 2003), SEGUE (Yanny et al. 2009), and LAMOST (Cui et al. 2012; Deng et al. 2012) surveys. The lower limit of iron abundance $[\mathrm{Fe} / \mathrm{H}],{ }^{1}$ which is often used as a proxy to the stellar metallicity, of the stars ever observed is -4.71 (SDSS J102915 + 172927; Caffau et al. 2011b) for carbon-normal stars. Some

1 The number abundance ratio of an element A to B is conventionally given with

$$
[\mathrm{A} / \mathrm{B}]=(A(\mathrm{~A})-A(\mathrm{~B}))-\left(A_{\odot}(\mathrm{A})-A_{\odot}(\mathrm{B})\right),
$$

where $A(\mathrm{~A})$ is the absolute abundance defined as

$$
A(\mathrm{~A})=12+\log \left(y_{\mathrm{A}}\right)
$$

and $y_{\mathrm{A}}$ is the number fraction of $\mathrm{A}$ relative to hydrogen nuclei. 
of the so-called carbon-enhanced metal-poor (CEMP) stars have even smaller fractions of $\mathrm{Fe}([\mathrm{Fe} / \mathrm{H}]<-5)$, but they have enhanced carbon abundances $[\mathrm{C} / \mathrm{Fe}]>0.7$ (Beers \& Christlieb 2005; Aoki et al. 2007). Another approach is to measure the elemental abundance ratio of extremely metalpoor (EMP) stars with metallicities $[\mathrm{Fe} / \mathrm{H}]<-3$, which are considered to form from clouds enriched by a single or several Pop III SNe (Ryan et al. 1996; Cayrel et al. 2004). Ishigaki et al. (2018) showed that the elemental abundance ratios of EMP stars are best fit with the Pop III SN models with progenitor masses $M_{\text {PopIII }}<40 \mathrm{M}_{\odot}$.

This reverse-engineering assumes that the expanding ejecta of Pop III SNe is uniformly mixed, and the averaged elemental abundance ratio in all layers of the ejecta is used when fitting the stellar abundances. According to stellar evolution models, the SN ejecta should be initially stratified, where heavier elements, such as $\mathrm{Fe}$, are in the inner layers and lighter elements, such as $\mathrm{C}$, are in the outer layers. If the expanding ejecta maintains its radial structure, it is expected that the abundance of star-forming clouds enriched by the SNe and stars that eventually form might be different from that from uniform ejecta. Supposing that these stars inherit the same abundance from their parent clouds, a bias is expected between the elemental abundances of observed EMP stars and monolithic SN ejecta.

In this work, we investigate the effect of stratified SN ejecta on the elemental abundance ratio of the succeeding generations of stars with cosmological simulations. In our previous work Chiaki et al. (2018, hereafter C18), we found that the three-dimensional density and velocity structures around the MHs have large effects on the dynamical evolution of SN shells. We also found that, SN shells fall back into MHs that originally hosted Pop III stars and collapse again when the smaller explosion energy $E_{\mathrm{SN}}$ is smaller than the halo binding energy. If the explosion energy is larger, neighboring MHs can also be enriched by Pop III SNe. These two distinctive enrichment modes are called internal enrichment (IE) and external enrichment (EE). The ratio of lighter to heavier elements is expected to be different between in the two cases where the SN ejecta is stratified and uniform.

The bias of an elemental abundance pattern in a recollapsing cloud from that of uniform ejecta was studied by Ritter et al. (2015) and Sluder et al. (2016) in the IE case. They performed a high-resolution simulation of metal enrichment from a Pop III SN, following the metal dispersion with tracer particles. They found that the mass fraction of metals originally in the innermost hotter layers escaping from the MH is three times larger relative to the metals in the outer layers. This would result in an enhancement of $\sim 0.5$ dex the abundance ratio of lighter elements to heavier elements. However, they only considered one explosion energy $E_{\mathrm{SN}}=1 \mathrm{~B}$ $\left(1 \mathrm{~B}=10^{51} \mathrm{erg}\right)$. The shell evolution and the resulting elemental abundance ratio of recollapsing clouds are expected to depend on $E_{\mathrm{SN}}$. SNe with higher explosion energies $(\sim 10$ $\mathrm{B}$; hypernovae) have been observed, accompanied with longduration gamma-ray bursts (Iwamoto et al. 1998). The elemental abundances of some EMP stars are best fit with hypernova moedels (Tominaga et al. 2014). This motivates us to consider higher explosion energies. In addition, they did not consider the radial distribution of individual heavy elements. To quantitatively predict the difference between the elemental abundances of clouds enriched by uniform and stratified ejecta, it is essential to consider radial distribution of each heavy element. Here we run simulations with a wide range of explosion energies $E_{\mathrm{SN}}=0.7-10 \mathrm{~B}$ with ejecta models taken from SN nucleosynthesis calculations that give an accurate radial distribution of heavy elements for each explosion energy.

Due to computational limits, we start the simulations at a time when the ejecta has expanded up to a radius $R_{\text {sh }} \simeq 3$ pc. The corresponding elapsed time from the $\mathrm{SN}$ explosion is $9.0 \times 10^{3} \mathrm{yr}\left(R_{\mathrm{sh}} / 3 \mathrm{pc}\right)^{5 / 2}\left(E_{\mathrm{SN}} / 1 \mathrm{~B}\right)^{-1 / 2}$ (McKee \& Ostriker 1977). Unfortunately, the mixing efficiency of ejecta in the early phase $(<1000 \mathrm{yr})$ is unknown. During the shock propagation in the stellar mantle, the layers in ejecta with different mean molecular weights can mix with each other between their boundaries because of Rayleigh-Taylor (RT) instabilities (Joggerst, Almgren \& Woosley 2010). Observations of the young ( 1000 yr) SN remnant (SNR) Cas A showed a knotty elemental distribution (Douvion et al. 2001), and X-ray/ $\gamma$-ray observations also showed that the ejecta of SN 1987A is partially mixed (Dotani et al. 1987). These observations suggest that the mixing state is somewhere between fully stratified and mixed. We therefore run simulations in two extreme cases, stratified and uniform ejecta. Our results give the upper and lower limits of its effect on the elemental abundances of the clouds that host second-generation stars.

The structure of this paper is as follows. In Section 2, we describe our numerical methods. We present our results in Section 3 and compare them with the elemental abundance in EMP stars in Section 4. Finally, we conclude in Section 5. Throughout this paper, the simulations are performed in comoving coordinates, but we quote proper values unless otherwise specified.

\section{NUMERICAL MODELS}

In this work, we use basically the same numerical method as in C18. We briefly summarize our basic setup in Sections 2.1-2.2 and detail the updated methods in Section 2.3.

\subsection{Simulation setup}

We utilize the SPH/N-body hydrodynamics code GADGET2 (Springel 2005), while considering non-equilibrium chemistry and cooling. The hydrodynamics of the gas component is solved with the standard SPH scheme with a fixed number of neighboring particles $N_{\mathrm{ngb}}=64 \pm 8$. To alleviate spurious surface tension on contact discontinuities (Saitoh \& Makino 2013; Hopkins 2013; Wadsley, Keller \& Quinn 2017), we adopt the shared timestep strategy, where the physical variables of all SPH particles are updated with a global timestep (Saitoh \& Makino 2009). The timestep is calculated as $\min _{i}\left\{\Delta t_{\mathrm{acc}, i}, \Delta t_{\mathrm{cou}, i}\right\}$, where $\Delta t_{\mathrm{acc}, i}$ and $\Delta t_{\mathrm{cou}, i}$ are the acceleration and Courant timescales of a gas particle $i$, respectively.

We solve the chemical networks of 53 reactions for 15 species, $\mathrm{e}^{-}, \mathrm{H}^{+}, \mathrm{H}, \mathrm{H}^{-}, \mathrm{H}_{2}^{+}, \mathrm{H}_{2}, \mathrm{D}^{+}, \mathrm{D}, \mathrm{D}^{-}, \mathrm{HD}^{+}$, $\mathrm{HD}, \mathrm{He}^{2+}, \mathrm{He}^{+}, \mathrm{He}$, and $\mathrm{HeH}^{+}$. Our chemical model includes collisional ionization/recombination of $\mathrm{H} / \mathrm{He}$ and the $\mathrm{H}^{-}$-process, $\mathrm{H}_{2}^{+}$-process, and three-body reactions for the formation of molecular hydrogen. We then calculate the 


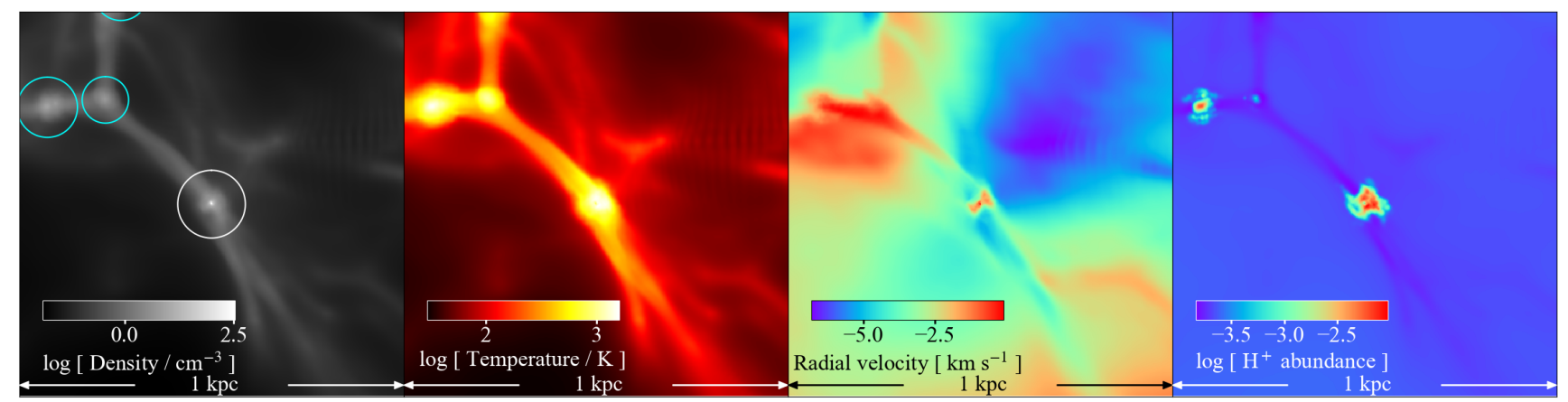

Figure 1. Density-weighted projection of density, temperature, and radial velocity relative to the central Pop III star, and $\mathrm{H}$ II abundance relative to hydrogen nuclei at the end of the lifetime of the progenitor star with mass $M_{\text {PopIII }}=25 \mathrm{M}_{\odot}$, just before its supernova explosion. The white and cyan circles depict the virial radii of the Pop III hosting halo which is eventually internally enriched and halos which will merge and be externally enriched for an explosion energy $E_{\mathrm{SN}}=10 \mathrm{~B}$.

cooling rates of inverse Compton, bremsstrahlung, ionization/recombination and collisional excitation of $\mathrm{H} / \mathrm{He}$, and ro-vibrational transition of $\mathrm{H}_{2} / \mathrm{HD}$ molecules. We here ignore $\mathrm{C}$ and $\mathrm{O}$ fine-structure cooling, which is dominated by HD cooling for metallicities $[\mathrm{C} / \mathrm{H}] \lesssim-3$ (Omukai et al. 2005; Jappsen et al. 2007).

We follow the dispersion of metals with Lagrangian tracer particles. The velocity $\boldsymbol{v}_{j}$ of a tracer particle $j$ are interpolated from the velocity $\boldsymbol{v}_{i}$ of neighboring SPH particles as

$$
\boldsymbol{v}_{j}=\sum_{i} \frac{m_{i}}{\rho_{i}} \boldsymbol{v}_{i} W\left(r_{i j}, \tilde{h}_{j}\right)
$$

where $m_{i}$ and $\rho_{i}$ are the mass and density of a gas particle $i$, respectively, and $W\left(r_{i j}, \tilde{h}_{j}\right)$ is the cubic-spline kernel function of the distance $r_{i j}$ between particles $i$ and $j$ (Springel 2005). The smoothing length $\tilde{h}_{j}$ is estimated so that a sphere with the radius $\tilde{h}_{j}$ contains four neighboring SPH particles. The number of neighboring particles for the velocity evaluation is smaller than the density evaluation to capture steep velocity gradients around SN shocks.

\subsection{Initial conditions}

We create initial conditions for a cosmological zoom-in simulation with the MUSIC code (Hahn \& Abel 2011). We initialize the simulation at $z_{\text {ini }}=99$ with cosmological parameters taken from Planck Collaboration et al. (2016). First, we run a dark matter simulation with $512^{3}$ particles in a $10 h^{-1}$ Mpc (comoving) periodic box. We refine dark matter particles around MHs identified with a friends-of friends (FOF) algorithm. Then, we restart the simulation from $z_{\text {ini }}$ including baryons. The minimum mass of dark matter and baryon particles are $m_{\mathrm{p}, \text { gas }}=4.45 \mathrm{M}_{\odot}$ and $m_{\mathrm{p}, \mathrm{dm}}=16.5 \mathrm{M}_{\odot}$, respectively. A MH forms at redshift $z_{\text {form }}=28.5$ with a virial radius $R_{\mathrm{vir}}=70.1 \mathrm{pc}$ and mass $M_{\mathrm{vir}}=3.33 \times 10^{5} \mathrm{M}_{\odot}$. We cut out a spherical region with a radius $2.5 \mathrm{kpc}$ centered on the center-of-mass of the $\mathrm{MH}$, which contains $9,994,502$ dark matter particles and 9,972,070 gas particles.

When the maximum density reaches $n_{\mathrm{H}, \mathrm{cen}}=10^{3} \mathrm{~cm}^{-3}$, we put a star particle with a mass $M_{\text {PopIII }}=25 \mathrm{M}_{\odot}$ at the center-of-mass of a region with densities $>n_{\mathrm{H}, \mathrm{cen}} / 3$. We solve radiation transport from the star with a scheme of Susa
(2006). The emission rate of hydrogen ionization photons is set to $Q(\mathrm{H})=7.58 \times 10^{48} \mathrm{~s}^{-1}$ (Schaerer 2002). Fig. 1 shows the density, temperature, and radial velocity of the $\mathrm{MH}$, and $\mathrm{H}$ II abundance relative to hydrogen nuclei at the end of the lifetime of the star $t_{\text {life }}=6.46 \mathrm{Myr}$, corresponding to a redshift $z=27.3$. The virial mass and radius of the halo grow to $M_{\mathrm{vir}}=5.11 \times 10^{5} \mathrm{M}_{\odot}$ and $R_{\mathrm{vir}}=87.6 \mathrm{pc}$, respectively, through smooth mass accretion. The gas is partially ionized within $\sim 1$ pc from the star, where density, temperature, and $\mathrm{H}^{+}$abundance are $n_{\mathrm{H}} \sim 300 \mathrm{~cm}^{-3}, T \sim 5000 \mathrm{~K}$, and $y\left(\mathrm{H}^{+}\right) \sim 0.1$, respectively. The radius of the $\mathrm{H}$ II region is smaller than the halo virial radius because the expansion of ionizing front is halted by the gas accretion onto the $\mathrm{MH}$ (see also Kitayama et al. 2004). ${ }^{2}$

Note that the radius of the ionized region in this simulation is smaller than the Strömgren radius $R_{\mathrm{St}}=$ $36\left(Q(\mathrm{H}) / 8 \times 10^{48} \mathrm{~s}^{-1}\right)\left(n_{\mathrm{e}} / 1 \mathrm{~cm}^{-3}\right)^{-2 / 3} \mathrm{pc}$ because of the resolution limit as we discuss in Section 4.2.1. Also, we here consider the formation of a single Pop III star in the $\mathrm{MH}$ although the fragmentation of accretion disks can lead to multiple Pop III star formation (e.g., Turk et al. 2009; Clark et al. 2011; Greif et al. 2012). In addition, we prevent star formation in the other MHs by switching off gas cooling in dense regions with $>10^{3} \mathrm{~cm}^{-3}$ at distances $>100 \mathrm{pc}$ from the central MH. Lastly, we do not consider a relative velocity offset between dark matter and baryons ("streaming velocity"). This would play an important role in the structure formation and star formation (Chiou et al. 2018, 2019; Druschke et al. 2019). The validity of these assumptions is discussed in Section 4.2.3.

\subsection{Supernova models}

From the snapshot at $t_{\text {life }}$ (Fig. 1), we run four simulations with explosion energies $E_{\mathrm{SN}}=0.7,1,5$, and $10 \mathrm{~B}$ $\left(1 \mathrm{~B}=10^{51} \mathrm{erg}\right)$, hereafter called as E0.7, E1, E5, and E10, respectively. We replace the star particle with a Pop III remnant particle with a mass $M_{\text {rem }}$ (Table 1) and uniformly inject the explosion energy $E_{\mathrm{SN}}$ to central $200 \mathrm{SPH}$ particles

\footnotetext{
2 The simulation is the same as the MH1-C25 run in C18 until the
} SN explosion occurs. 


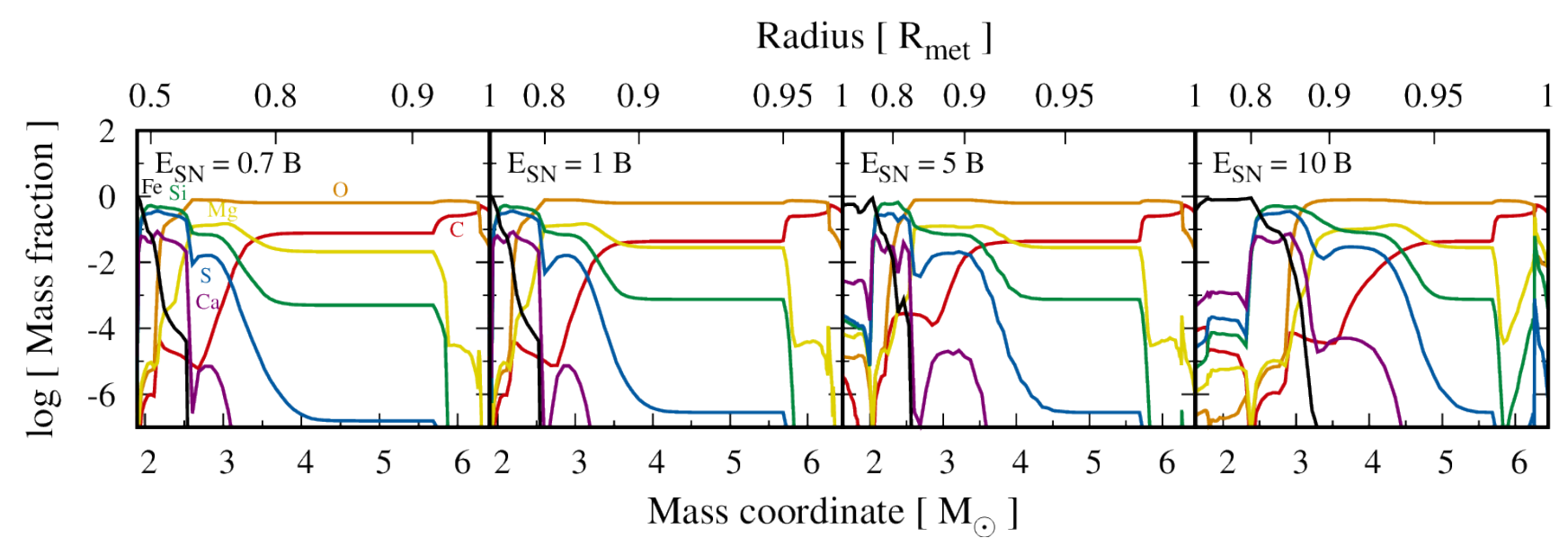

Figure 2. Mass fraction of major elements $\mathrm{C}$ (red), O (orange), $\mathrm{Mg}$ (yellow), Si (green), S (blue), Ca (purple), and Fe (black) as a function of enclosed mass and radius of ejecta for our progenitor models with a mass $M_{\mathrm{PopIII}}=25 \mathrm{M}_{\odot}$ and explosion energy $E_{\mathrm{SN}}=0.7$, 1,5 , and $10 \mathrm{~B}\left(1 \mathrm{~B}=10^{51} \mathrm{erg}\right)$. We plot the results of nucleosynthesis calculations of Tominaga et al. (2014) only in the CO core (see text). The top $x$-axis shows the spatial scale of the ejecta normalized by the radius $R_{\text {met }}$ of the CO core.

Table 1. SN models

\begin{tabular}{cccccccccc}
\hline${ }^{1}$ Run & $\begin{array}{c}{ }^{2} E_{\mathrm{SN}} \\
{[\mathrm{B}]}\end{array}$ & $\begin{array}{c}{ }^{3} M_{\text {rem }} \\
{\left[\mathrm{M}_{\odot}\right]}\end{array}$ & $\begin{array}{c}{ }^{4} M_{\text {met }} \\
{\left[\mathrm{M}_{\odot}\right]}\end{array}$ & $\begin{array}{c}{ }^{5} R_{\text {met }} \\
{\left[10^{-6} \mathrm{pc}\right]}\end{array}$ & $\begin{array}{c}{ }^{6} R_{\text {eje }} \\
{\left[10^{-6} \mathrm{pc}\right]}\end{array}$ & $\begin{array}{c}{ }^{7} M_{\mathrm{C}} \\
{\left[\mathrm{M}_{\odot}\right]}\end{array}$ & $\begin{array}{c}{ }^{8} M_{\mathrm{O}} \\
{\left[\mathrm{M}_{\odot}\right]}\end{array}$ & $\begin{array}{c}{ }^{9} M_{\mathrm{Fe}} \\
{\left[\mathrm{M}_{\odot}\right]}\end{array}$ & ${ }^{10}[\mathrm{C} / \mathrm{Fe}]_{0}$ \\
\hline \hline E0.7 & 0.7 & 1.90 & 4.51 & 1.14 & 19.4 & 0.384 & 2.61 & 0.0689 & 0.48 \\
E1 & 1 & 1.90 & 4.55 & 1.89 & 51.6 & 0.331 & 2.58 & 0.0885 & 0.31 \\
E5 & 5 & 1.62 & 4.83 & 5.79 & 47.6 & 0.329 & 2.61 & 0.271 & -0.18 \\
E10 & 10 & 1.62 & 4.83 & 8.42 & 66.7 & 0.285 & 2.23 & 0.699 & -0.65 \\
\hline
\end{tabular}

Note - (1) ID of runs. (2) Explosion energy. (3-4) Mass of a compact remnant and CO core. (5-6) Radius of CO core and ejecta. (7-9) Mass of synthesized C, O, and Fe. (10) Average abundance ratio $[\mathrm{C} / \mathrm{Fe}]$ in the $\mathrm{CO}$ core.

in a sphere with a radius $R_{\text {eje,sim }}=2.77$ pc. We deposit all of the explosion energy as thermal energy.

In this region, we insert $10^{6}$ metal particles, and the elemental mass fractions of each particle are remapped from one-dimensional stellar evolution/nucleosynthesis models of Tominaga et al. (2014). In their models, heavier elements than $\mathrm{He}$ are contained in only a small part of the ejecta. The metal-rich region with $X(\mathrm{H})+X(\mathrm{He})<0.95$ (hereafter called "CO core") lies only within the radius $R_{\text {met }}$, at least $\sim 4 \%$ of entire ejecta radius $R_{\text {eje }}$ (Table 1 ), where $X(\mathrm{M})$ is the mass fraction of an element $M$. If we include the all part of ejecta, the velocity of metal particles in the CO core would be interpolated from only $200 \times 0.04^{3}=0.01 \mathrm{SPH}$ particles (Table 1). Therefore, we consider only the CO core in our simulations. Fig. 2 shows the mass fractions of major elements in the $\mathrm{CO}$ core calculated by Tominaga et al. (2014).

We note that $2.34 \%, 13.1 \%, 13.1 \%$, and $14.8 \%$ of $\mathrm{C}$ is produced in the outer region containing mainly primordial elements (called "hydrogen envelope") for E0.7, E1, E5, and E10, respectively. This means $0.01-0.07$ dex loss of carbon mass. We also note that only $1.18 \times 10^{-5}, 2.09 \times 10^{-4}, 3.65 \times$ $10^{-4}$, and $8.66 \times 10^{-4}$ of $\mathrm{N}$ is produced in the CO core. Thus, even without the hydrogen envelope, the radial velocity of the $\mathrm{CO}$ core is not overestimated because of the deceleration from the swept up materials.

Table 1 shows the $\mathrm{CO}$ core mass $M_{\text {met }}$ and $\mathrm{C}$, $\mathrm{O}$, and Fe mass $\left(M_{\mathrm{C}}, M_{\mathrm{O}}\right.$, and $M_{\mathrm{Fe}}$, respectively). The dominant element is $\mathrm{O}$ for all $E_{\mathrm{SN}}$. The mass of lighter elements decreases while the mass of heavier elements increases with increasing $E_{\mathrm{SN}}$. The elemental abundance ratio of the uniform ejecta, which is often used when fitting EMP stars, are calculated from the total mass of each element produced in the ejecta. We hereafter attach the index ' 0 ' to depict the average elemental abundance ratios. In our normal CCSN model, the average carbon-to-iron abundance ratio $[\mathrm{C} / \mathrm{Fe}]_{0}$ increases from -0.65 for E10 to 0.48 for E0 . 7 , below the definition of CEMP stars $([\mathrm{C} / \mathrm{Fe}]=0.7)$. Note that $80 \%$ of EMP stars are C-enhanced stars with metallicities $[\mathrm{Fe} / \mathrm{H}]<-4.0$ (Yoon et al. 2018; Norris \& Yong 2019). We discuss the metal enrichment scenario from a different SN model which can explain C-enhanced star formation in Section 4.2.4.

We terminate the simulations at the time $t_{\text {recol }}$ when the maximum density of an enriched gas cloud reaches $n_{\mathrm{H}, \mathrm{cen}}=$ $10^{3} \mathrm{~cm}^{-3}$. We compare the elemental abundance within half of a Jeans length

$$
R_{\mathrm{J}}=\frac{1}{2}\left(\frac{\pi c_{\mathrm{s}}^{2}}{G \rho}\right)^{1 / 2}
$$




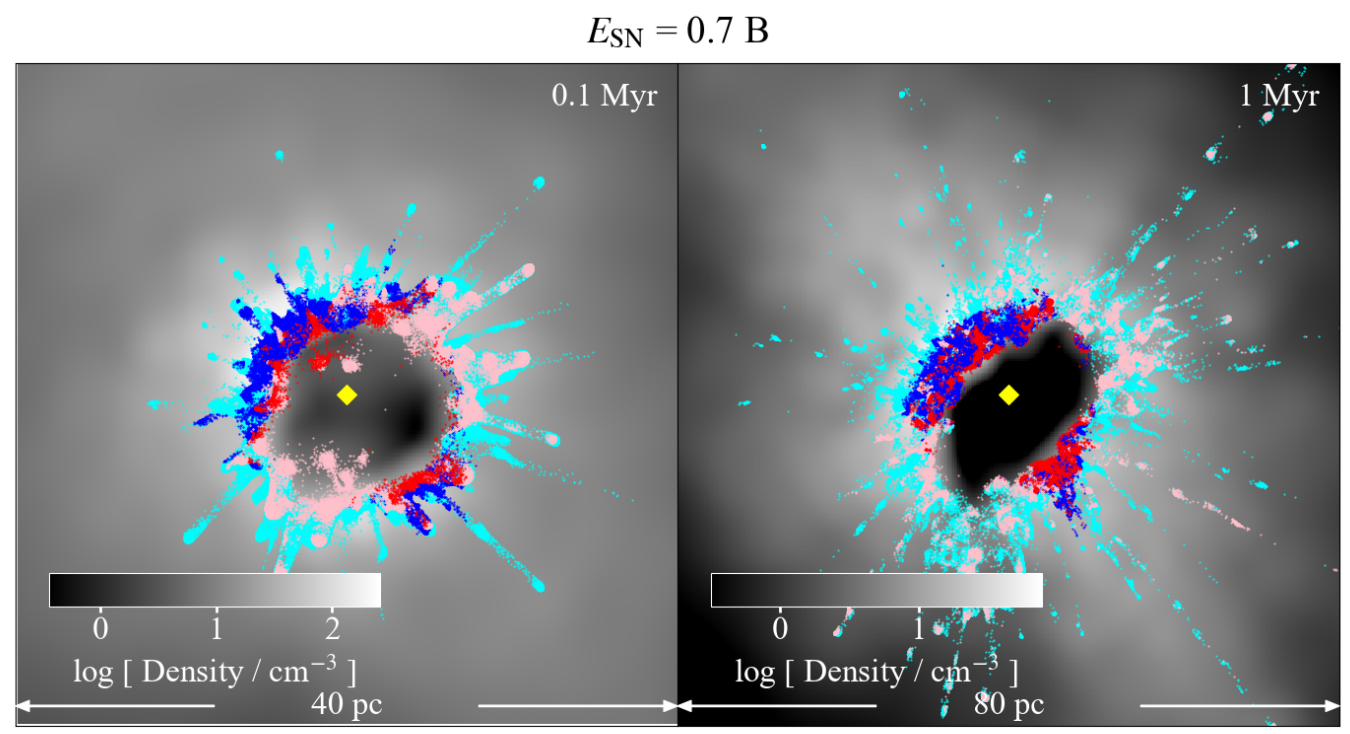

Figure 3. Slices of density $0.1 \mathrm{Myr}$ (left panel) and $1 \mathrm{Myr}$ (right panel) after the supernova for an explosion energy $E_{\mathrm{SN}}=0.7 \mathrm{~B}$. The panels are centered on the Pop III remnant (yellow diamond). We over-plot the location of lighter and heavier element dominant metal particles with $X(\mathrm{C})>X(\mathrm{Fe})$ (blue dots) and with $X(\mathrm{Fe})>X(\mathrm{C})$ (red dots), respectively, in a slab with the depth of 0.1 times the window size, where $X(\mathrm{M})$ is the mass fraction of metal element M. The dots with darker colors represent the particles which eventually fall back into the recollapsing cloud center $\left(r<R_{\mathrm{J}}\right)$.

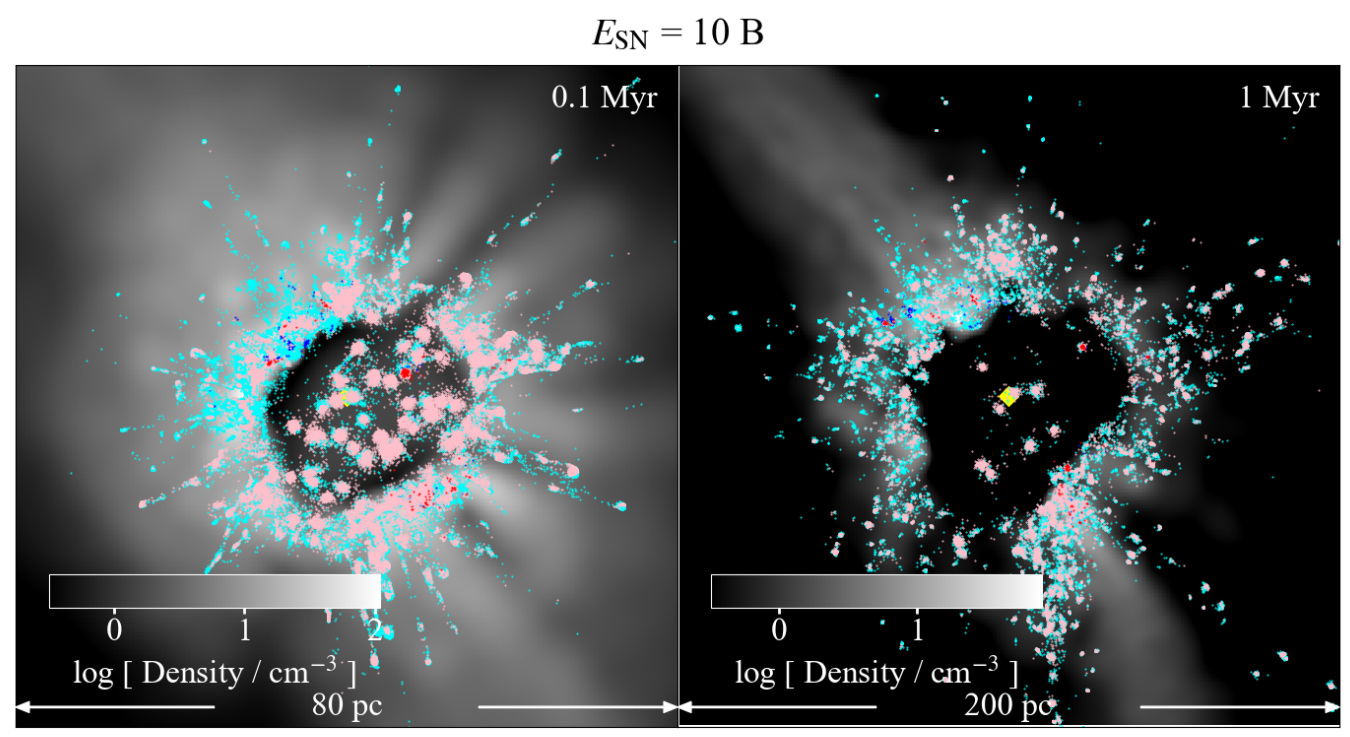

Figure 4. Same as Fig. 3 but for an explosion energy $E_{\mathrm{SN}}=10 \mathrm{~B}$.

with the abundances from uniformly mixed ejecta.

\section{RESULTS}

\subsection{Overview}

Figs. 3 and 4 show the gas density at 0.1 and 1 Myr after the SN explosion for E0.7 and E10. The blue and red dots depict the distribution of metal particles with $X(\mathrm{C})>X(\mathrm{Fe})$ and $X(\mathrm{C})<X(\mathrm{Fe})$, respectively. In all progenitor models, a part of ejecta falls back mainly along the cosmological filaments after the SN shells lose its thermal energy through radiative cooling in the dense contact surfaces with the filaments (Fig. 1). The gas falling back into the $\mathrm{MH}$ begins to collapse again, i.e., internal enrichment (IE) occurs at $t_{\text {recol }}=5.69-37.2$ Myr for different explosion energies (Table 2). The darker colors in Figs. 3 and 4 depict the particles which eventually fall back into the MH. These particles are confined in the contact surfaces.

Fig. 5 shows the radial distribution of density, $A(\mathrm{C})$, 


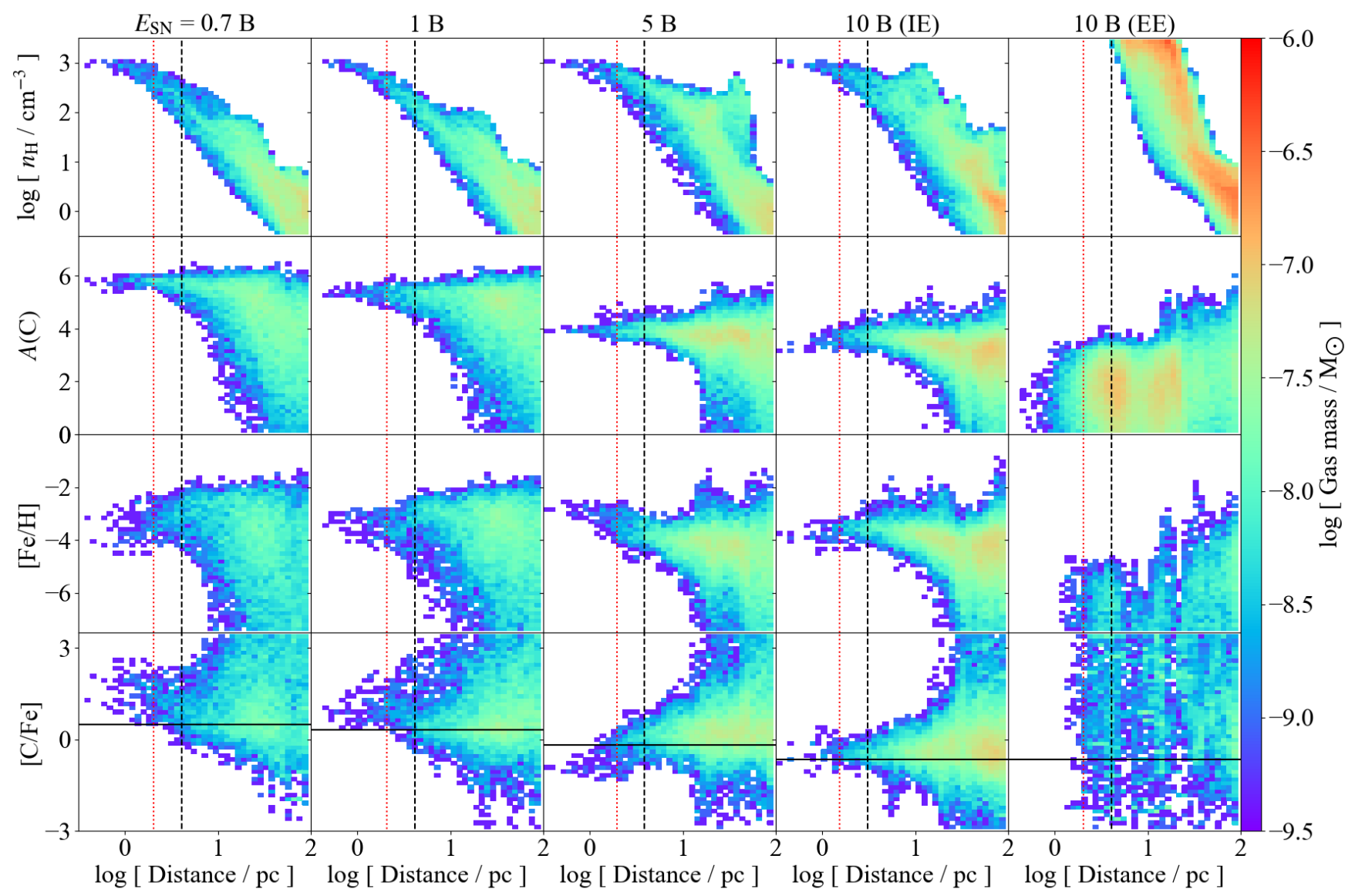

Figure 5. Two-dimensional histogram of density, $A(\mathrm{C}),[\mathrm{Fe} / \mathrm{H}]$, and $[\mathrm{C} / \mathrm{Fe}]$ as a function of radius from the density maximum at the time when the density of the enriched clouds reaches $10^{3} \mathrm{~cm}^{-3}$. The horizontal black solid lines show [C/Fe] with uniform ejecta. The vertical black dashed and red dotted lines show half of the Jeans length of the recollapsing clouds and resolution limit, respectively (see text).

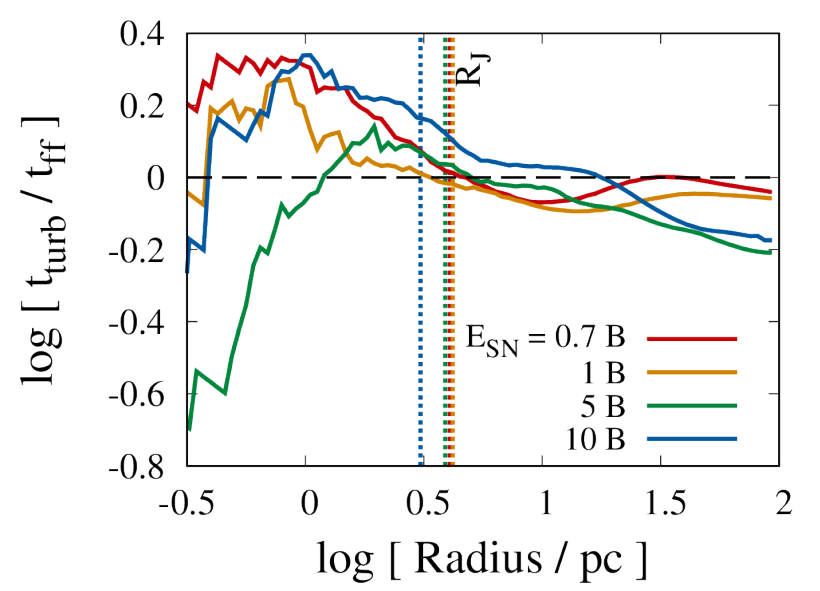

Figure 6. Ratio of the timescale of turbulent mixing $t_{\text {turb }}$ to free-fall time $t_{\mathrm{ff}}$ as a function of radius at the time $t_{\text {recol }}$ when the density of recollapsing clouds reaches $10^{3} \mathrm{~cm}^{-3}$ (solid curves). The dotted lines show half of the Jeans length $R_{\mathrm{J}}$. The colors depict explosion energies with $E_{\mathrm{SN}}=0.7 \mathrm{~B}$ (red), $1 \mathrm{~B}$ (orange), 5 B (green), and $10 \mathrm{~B}$ (blue).
$[\mathrm{Fe} / \mathrm{H}]$, and $[\mathrm{C} / \mathrm{Fe}]$ of the gas particles as a function of distance from the density maximum of the enriched clouds. We also show half of the Jeans length $R_{\mathrm{J}}$ (black dashed line) and the resolution limit defined as twice the smoothing length (red dotted line). The carbon and iron abundances show large deviations at distances $>10 \mathrm{pc}$ because of the non-uniform metal mixing in the voids. Toward the higher density region at distances $<3 \mathrm{pc}$, the metal abundances converge. In Fig. 6 we compare the timescale for metal mixing due to turbulence, $t_{\text {turb }}=R / \sigma_{v}$, with the free-fall timescale, $t_{\mathrm{ff}}=(3 \pi / 32 G \bar{\rho})^{1 / 2}$, where $\sigma_{v}$ and $\bar{\rho}$ are the velocity dispersion and mean density within a radius $R$, respectively. Within $R \lesssim 3$ pc the mixing timescale becomes longer than the collapsing timescale, which indicates that further metal mixing does not occur as pointed out by earlier studies (Smith et al. 2015; Ritter et al. 2015; Chiaki \& Wise 2019).

We measure the average abundance of each element within $R_{\mathrm{J}} \sim 3 \mathrm{pc}$, regarding it as the characteristic abundance of the clouds. The red and black curves in Fig. 7 show the elemental abundance ratios $[\mathrm{M} / \mathrm{Fe}]$ for the stratified and uniform ejecta, respectively. The difference $\Delta[\mathrm{M} / \mathrm{Fe}]$ of these abundances is within \pm 0.4 dex for all elements for all progen- 


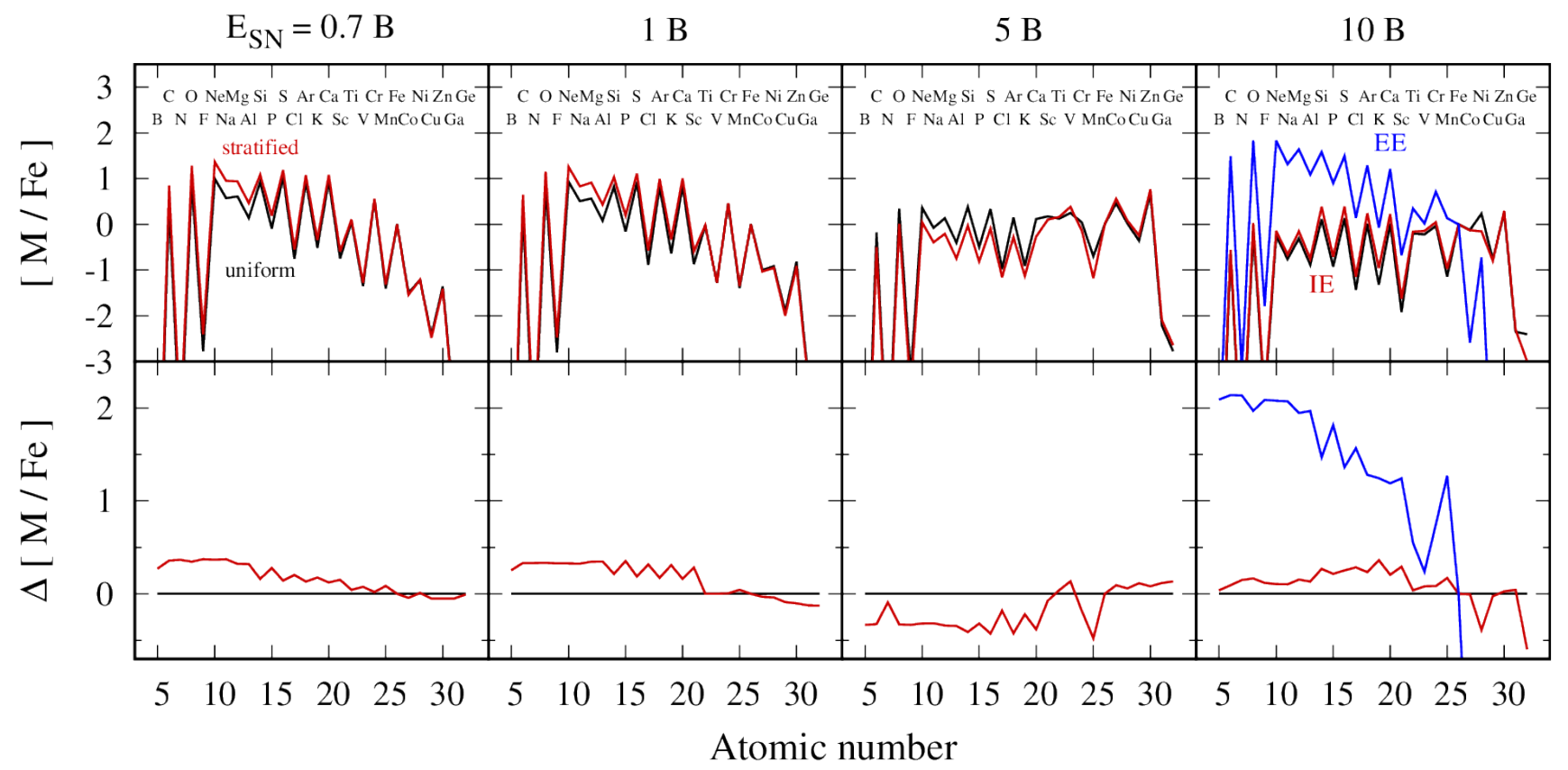

Figure 7. Abundance ratios of element $\mathrm{M}$ from $\mathrm{B}$ to Ge relative to iron in the internally (red) and externally (blue) enriched clouds from the stratified ejecta for explosion energies $E_{\mathrm{SN}}=0.7 \mathrm{~B}, 1 \mathrm{~B}, 5 \mathrm{~B}$, and $10 \mathrm{~B}$ from left to right. The black curves show the elemental abundance of the uniform ejecta.

itor models (bottom panels of Fig. 7). We can conclude that the stratified structures of the ejecta hardly affect on the elemental abundance ratio of the internally enriched clouds, which we will further analyze in Section 3.2.1 (i).

For E10, metals reach the neighboring halo, and external enrichment (EE) occurs (Fig. 8) as well as IE. The blue curve of Fig. 9 shows that only the outer layers of ejecta with mass coordinates $M_{r}>2.5 \mathrm{M}_{\odot}$ reach the neighboring halo. Since these layers mainly contain lighter elements (bottom panel of Fig. 7), the relative abundances of lighter elements $(<\mathrm{Ca})$ are enhanced more than $\Delta[\mathrm{M} / \mathrm{Fe}]>1$. This indicates that stars with the C-enhanced abundance $([\mathrm{C} / \mathrm{Fe}]=1.49)$ can form in externally enriched clouds from normal CCSNe.

\subsection{Evolution of SN shells}

\subsubsection{Internal enrichment}

\section{(i) General behavior}

In the case of IE, there is only a slight difference $( \pm 0.4$ dex) in the elemental abundance ratio between the clouds enriched by the stratified and uniform ejecta. Fig. 9 shows the mass fraction $f_{\mathrm{enr}}\left(M_{\mathrm{r}}\right)$ of metals falling back into the $\mathrm{MH}$ as a function of initial mass mass coordinate $M_{\mathrm{r}}$ of the ejecta. For all $E_{\mathrm{SN}}, f_{\mathrm{enr}}\left(M_{\mathrm{r}}\right)$ is almost constant against $M_{\mathrm{r}}$. Therefore, the ratio between lighter and heavier elements does not significantly deviate from that for the uniform ejecta.

In this section, we interpret this property by reviewing the evolution of SN shells. First, in the Sedov-Taylor (ST) phase, the shell evolves adiabatically because the timescale of radiative cooling is longer than the elapsed time. Then the shell enters the pressure-driven snowplough (PDS) phase, in

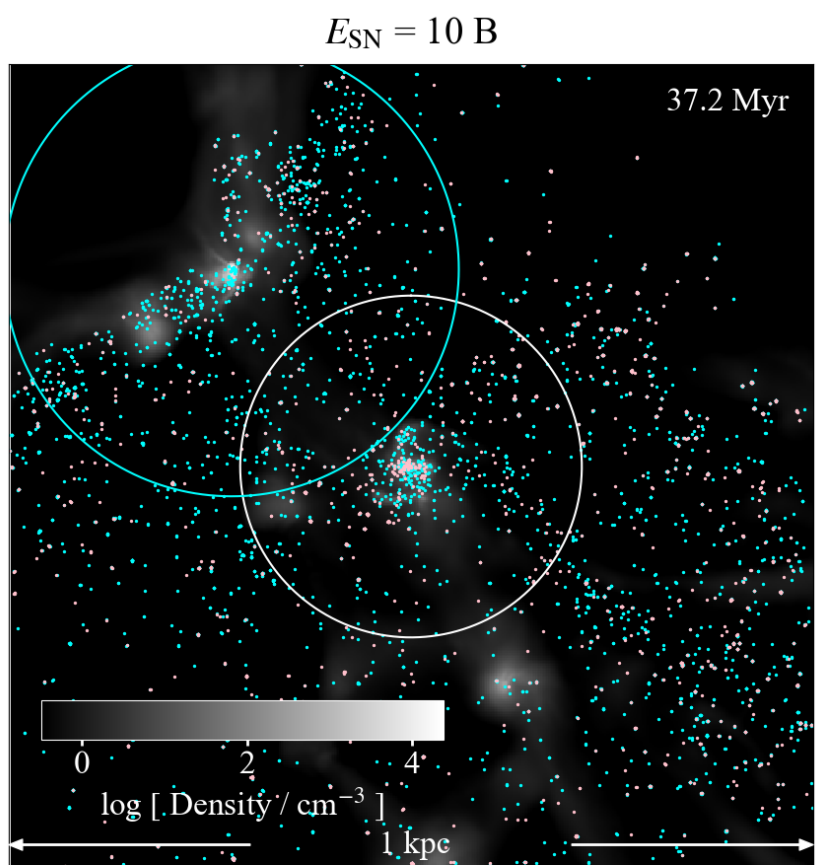

Figure 8. Density-weighted projection of density for an explosion energy $E_{\mathrm{SN}}=10 \mathrm{~B}$ and location of C-dominated (cyan) and Fe-dominated (pink) metal particles. We plot every 100 particles. The white and cyan circles denote virial radii of the Pop III hosting halo and externally enriched halo. 


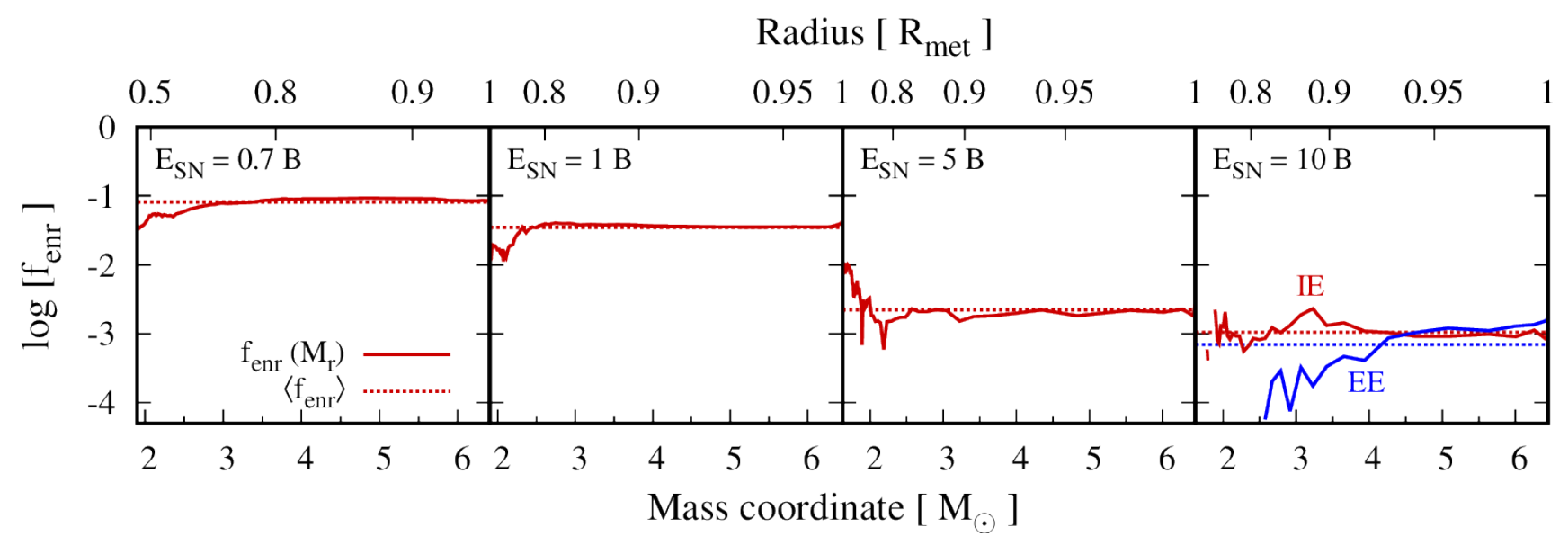

Figure 9. Radial profile of mass fraction $f_{\text {enr }}$ of metals incorporated into the subsequent star-forming clouds at the time when the gas density reaches $10^{3} \mathrm{~cm}^{-3}$. The positions of metals are traced back to the initial mass coordinate and radius of ejecta (the same axes as Fig. 2). The red and blue curves depict the results for internal and external enrichment, respectively. We show $f_{\text {enr }}\left(M_{\mathrm{r}}\right)$ as a function of mass coordinate $M_{\mathrm{r}}$ (solid curves) and their mass-weighted average $\left\langle f_{\text {enr }}\right\rangle$ among radial bins (dotted curves).

Table 2. Metallicity in enriched clouds

\begin{tabular}{cccccccccccc}
\hline $\begin{array}{c}{ }^{1} E_{\mathrm{SN}} \\
{[\mathrm{B}]}\end{array}$ & $\begin{array}{c}{ }^{2} t_{\text {recol }} \\
{[\mathrm{Myr}]}\end{array}$ & ${ }^{3} z_{\text {recol }}$ & ${ }^{4}$ enrich. & $\begin{array}{c}{ }^{5} M_{\text {vir }} \\
{\left[\mathrm{M}_{\odot}\right]}\end{array}$ & $\begin{array}{c}{ }^{6} R_{\text {vir }} \\
{[\mathrm{pc}]}\end{array}$ & $\begin{array}{c}{ }^{7} M_{\mathrm{cl}} \\
{\left[\mathrm{M}_{\odot}\right]}\end{array}$ & $\begin{array}{c}{ }^{8} R_{\mathrm{J}} \\
{[\mathrm{pc}]}\end{array}$ & ${ }^{9} A(\mathrm{C})$ & ${ }^{10}[\mathrm{Fe} / \mathrm{H}]$ & ${ }^{11}[\mathrm{C} / \mathrm{Fe}]$ & ${ }^{12} \Delta[\mathrm{C} / \mathrm{Fe}]$ \\
\hline \hline 0.7 & 5.69 & 26.4 & IE & $7.25 \times 10^{5}$ & 102 & $1.76 \times 10^{3}$ & 4.08 & 6.32 & -2.95 & 0.84 & 0.36 \\
1.0 & 7.30 & 26.2 & IE & $7.82 \times 10^{5}$ & 105 & $1.81 \times 10^{3}$ & 4.19 & 5.86 & -3.21 & 0.64 & 0.33 \\
5.0 & 24.7 & 23.9 & IE & $2.02 \times 10^{6}$ & 158 & $2.01 \times 10^{3}$ & 3.89 & 4.56 & -3.36 & -0.50 & -0.33 \\
10.0 & 37.2 & 22.5 & IE & $4.13 \times 10^{6}$ & 212 & $1.39 \times 10^{3}$ & 3.06 & 4.32 & -3.55 & -0.56 & 0.09 \\
& & & EE & $9.73 \times 10^{6}$ & 282 & $1.40 \times 10^{5}$ & 4.09 & 2.48 & -7.44 & 1.49 & 2.14 \\
\hline
\end{tabular}

Note - (1) Explosion energy. (2) Time of recollapse from SN explosion. (3) Redshift of recollapse. (4) Enrichment mode. (5-6) Virial mass and radius of the $\mathrm{MH}$ at the time of recollapse. (7-8) Mass and half of the Jeans length of the recollapsing cloud. (9-11) Average carbon and iron abundances and relative abundance ratio $[\mathrm{C} / \mathrm{Fe}]$ of the recollapsing cloud. (12) Difference of $[\mathrm{C} / \mathrm{Fe}]$ between the stratified and uniform ejecta.

which the dense cooling shell is pushed by the pressure in a hot inner cavity. Because of the inertial force from the decelerating shell to the ISM, RT instabilities develops and induces the mixing of materials between the shell and ISM. After the internal cavity cools down through radiative cooling, the shell continues to expand with its momentum being conserved in the phase called momentum-conserving snowplough (MCS) phase (Ostriker \& McKee 1988). The shell eventually either dissolves into the ISM if the explosion energy is enough high or otherwise falls back into the $\mathrm{MH}$ (Ritter et al. 2012; Sluder et al. 2016; Smith et al. 2015; Chiaki \& Wise 2019)

Figs. 3 and 4 show that lighter and heavier elements are both concentrated and partly mixed in the dense cooling shells which have developed in the PDS phase. As depicted by the dots with darker colors, both lighter and heavier elements that eventually fall back into the $\mathrm{MH}$ are confined in the same region where the SN shell interacts with the dense cosmological filaments. Using the radius of the filaments $R_{\text {fil }}$ and the radius of the SN shell $R_{\text {sh }}$, we can estimate the fall back fraction as the ratio of the solid angles $f_{\mathrm{enr}}=\pi R_{\mathrm{fil}}^{2} / 4 \pi R_{\mathrm{sh}}^{2}$ (Ritter et al. 2015). From this, the abun- dances of $\mathrm{C}$ and Fe which fall back can be estimated as

$$
\begin{aligned}
A(\mathrm{C}) & =12+\log \left(\frac{f_{\mathrm{enr}} M_{\mathrm{C}} / \mu_{\mathrm{C}}}{X_{\mathrm{H}} M_{\mathrm{cl}}}\right) \\
{[\mathrm{Fe} / \mathrm{H}] } & =12+\log \left(\frac{f_{\mathrm{enr}} M_{\mathrm{Fe}} / \mu_{\mathrm{Fe}}}{X_{\mathrm{H}} M_{\mathrm{cl}}}\right)-A_{\odot}(\mathrm{Fe}),
\end{aligned}
$$

where $\mu_{\mathrm{M}}$ is the molecular weight of an element $\mathrm{M}\left(\mu_{\mathrm{C}}=12\right.$, $\left.\mu_{\mathrm{Fe}}=56\right)$ and $A_{\odot}(\mathrm{Fe})=7.50$ is the solar abundance of $\mathrm{Fe}$ (Asplund et al. 2009). The mass $M_{\mathrm{cl}}$ is defined as the Jeans mass of the recollapsing clouds. From these equations, the abundance ratio

$$
[\mathrm{C} / \mathrm{Fe}]=\left(A(\mathrm{C})-A_{\odot}(\mathrm{C})\right)-[\mathrm{Fe} / \mathrm{H}]
$$

is the same as that for the uniform ejecta

$$
[\mathrm{C} / \mathrm{Fe}]_{0}=\log \left(\frac{M_{\mathrm{C}} / \mu_{\mathrm{C}}}{M_{\mathrm{Fe}} / \mu_{\mathrm{Fe}}}\right)
$$

because the factor $\log f_{\text {enr }}$ is canceled out. Since the factor depends only on the geometry of the circumstellar medium, the fall back fraction of each elements is almost the same.

For E0.7 and E5, the shell radius in the direction of filaments is $R_{\mathrm{sh}}=20 \mathrm{pc}$ and $60 \mathrm{pc}$, and the radius of the dense part of the filaments is $R_{\mathrm{fil}}=10 \mathrm{pc}$ and $5 \mathrm{pc}$, respectively. Then, the enrichment fraction is $f_{\mathrm{enr}}=6.25 \times 10^{-2}$ 
and $1.74 \times 10^{-3}$. This is consistent with the average fall back fraction estimated from our simulations $\left(8.12 \times 10^{-2}\right.$ and $2.23 \times 10^{-3}$; dotted lines in Fig. 9). With the typical mass of recollapsing clouds $M_{\mathrm{cl}}=2000 \mathrm{M}_{\odot}$ (Table 2), the abundances can be estimated to be $(A(\mathrm{C}),[\mathrm{Fe} / \mathrm{H}])=$ $(6.12,-2.80)$ and $(4.50,-3.76)$ for E0 .7 and E5, respectively. These estimates are also consistent with the simulation results.

\section{(ii) Dependency of elemental abundance on $E_{\mathrm{SN}}$}

The difference of the abundance ratio of lighter to heavier elements between the stratified and uniform ejecta depends slightly on $E_{\mathrm{SN}}$. The bottom panels of Fig. 7 show that the ratio is higher than that from the uniform ejecta for lower explosion energies $E_{\mathrm{SN}} \leq 1 \mathrm{~B}(0.33<\Delta[\mathrm{C} / \mathrm{Fe}]<0.36)$ while similar or smaller for higher $E_{\mathrm{SN}} \geq 5 \mathrm{~B}(-0.33<$ $\Delta[\mathrm{C} / \mathrm{Fe}]<0.09)$. The shell evolution and elemental abundances in the enriched clouds mostly differ between the two cases with explosion energies $E_{\mathrm{SN}} \leq 1 \mathrm{~B}$ and $E_{\mathrm{SN}} \geq 5 \mathrm{~B}$, hereafter called low and high $E_{\mathrm{SN}}$, respectively. We compare these two cases in this section.

For low $E_{\mathrm{SN}}$, the MCS phase begins at $\sim 0.1 \mathrm{Myr}$ after the SN explosion. In the MCS phase, the contact surface of the shell in the direction of filaments is decelerated from the pressure of infalling gas along the filaments with a radial velocity $\sim 6 \mathrm{~km} \mathrm{~s}^{-1}$ (Fig. 1). In comparison, the hot inner part of the shell containing the heavier elements circumvents the dense contact surface and continues to expand in the direction of voids. Therefore, the abundance of Fe falling back into the cloud is slightly lower than the simple estimation of Eq. (4). As a result, the abundance ratio of lighter to heavier elements from the stratified ejecta becomes slightly larger than that from the uniform ejecta $(0.33<\Delta[\mathrm{C} / \mathrm{Fe}]<0.36)$. This is the consistent result with the high-resolution simulation of Ritter et al. (2015) and Sluder et al. (2016).

For high $E_{\mathrm{SN}}$, the shell reaches a larger distance $(\sim 60$ pc) and propagates into the less dense region $\left(\lesssim 1 \mathrm{~cm}^{-3}\right)$ than for low $E_{\mathrm{SN}}$ (Fig. 4). The pressure from the filaments on the contact surface is smaller and the outer part of the shell is decelerated less efficiently. The inner part of the shell moves radially without circumventing the contact surface. Therefore, the deviation of the abundance ratio between lighter and heavier elements falling back into the $\mathrm{MH}$ is smaller than with lower $E_{\mathrm{SN}}$. For E10, the elemental abundance ratio in the recollapsing cloud is consistent with that from the uniform ejecta $(\Delta[\mathrm{C} / \mathrm{Fe}]=0.09)$. However, for E5, the iron abundances are enhanced by $\sim 0.4$ dex. A part of the innermost layers that is initially in the direction to the void moves to the contact surface between the shell and filament because of the turbulence driven by the energetic explosion. This region contains $40.6 \%$ of the total iron mass that eventually falls back into the central MH. This results in the enhancement of iron relative to the elemental abundance of the uniform ejecta $(\Delta[\mathrm{C} / \mathrm{Fe}]=-0.33)$, suggesting that the hydrodynamics in the shell can induce a $\sim 0.3$ dex of variation in the elemental abundance ratio.

\subsubsection{External enrichment}

For the EE case, in general, although metals reach a neighboring halo, they are hardly mixed with the gas in the cen- tral star-forming core (Chen et al. 2017; Chiaki et al. 2018). If gas density in the halo already exceeds a threshold value $n_{\mathrm{H}, \mathrm{th}} \sim 10 \mathrm{~cm}^{-3}$, the metals only superficially enrich the envelope of the cloud due to the pressure gradient. The metals can penetrate into the cloud center only if the SN energy is sufficiently strong (Chen et al. 2017) or if the halo merges with other halos (Chiaki et al. 2018). For E10, metals reach a neighboring halo initially at a distance $D=418 \mathrm{pc}$ from the central MH. The halo merges with two other halos (cyan circles in Fig. 1) at $\sim 1$ Myr after the SN explosion and then EE occurs. At $t_{\text {recol }}=37.2 \mathrm{Myr}$, the merged cloud becomes closer to the central MH with $D=333$ pc (Fig. 8). The radius of the region with densities above $n_{\mathrm{H}, \mathrm{th}}$, which can trap metals, is $R_{\mathrm{cl}} \simeq 20$ pc (Fig. 5).

Only the outer layers of the ejecta with initial mass coordinates $M_{\mathrm{r}}>2.5 \mathrm{M}_{\odot}$ reach the neighboring halo. The fraction of the materials incorporated into the cloud is $f_{\text {enr }}\left(M_{\mathrm{r}}\right) \sim 10^{-3}$ at $M_{\mathrm{r}}>4 \mathrm{M}_{\odot}$ (the blue curve in Fig. 9). This fraction can be explained with the simple estimation used in Section 3.2.1 as $f_{\mathrm{enr}}=\pi R_{\mathrm{cl}}^{2} / 4 \pi D^{2}=9.01 \times 10^{-4}$. With the mass of the enriched cloud $M_{\mathrm{cl}}=1.40 \times 10^{5} \mathrm{M}_{\odot}$, we can estimate the $\mathrm{C}$ abundance of the enriched cloud to be $A(\mathrm{C})=2.30$ from Eq. (3), consistent with the simulation result $A(\mathrm{C})=2.48$ (Table 2). The abundance is much smaller than those in the case with IE by $\sim 2$ dex because the metals are diluted by the larger mass of pristine gas by two orders of magnitude than the typical cloud mass $\left(2000 \mathrm{M}_{\odot}\right)$. The cloud mass is much larger because the halo hosting the cloud grows through a set of mergers before EE occurs.

\section{DISCUSSION}

\subsection{Prediction of observations}

If low-mass stars $\left(M_{*}<0.8 \mathrm{M}_{\odot}\right)$ form in the enriched clouds, they can survive for the Hubble time (Smith et al. 2015; Chiaki \& Wise 2019). Then, if some of the stars are accreted onto our Milky Way halo or local dwarf galaxies through cosmic structure formation, they can be observed as EMP stars (Frebel \& Norris 2015, and references therein). The colored symbols in Fig. 10 show the distribution of the stars on the $A(\mathrm{C})-[\mathrm{Fe} / \mathrm{H}]$ plane. The closed and open symbols depict the abundances of stars forming in the internally and externally enriched clouds (hereafter called IE-stars and EE-stars), respectively. The small and large symbols depict the abundances for the uniform and stratified ejecta, respectively. Interestingly, the Fe abundances are shifted while $\mathrm{C}$ abundances hardly change. The mass of the outer layers is larger than that of the inner layers, where the fall back fraction fluctuates, and the mass-weighted average fraction $\left\langle f_{\text {enr }}\right\rangle$ is closer to the fraction $f_{\text {enr }}\left(M_{\mathrm{r}}\right)$ in the outer layers (Fig. 9).

For comparison, we also plot the $\mathrm{C}$ and $\mathrm{Fe}$ abundances of observed C-normal and C-enhanced stars with blue and red dots, respectively (taken from the SAGA database; Suda et al. 2008). The stars with below and above $[\mathrm{C} / \mathrm{Fe}]=0.7$ (dashed line) are defined as C-normal stars and CEMP stars, respectively (Aoki et al. 2007). C-normal stars are distributed around the line $[\mathrm{C} / \mathrm{Fe}]=0$ (dot-dashed line). CEMP stars with $[\mathrm{Fe} / \mathrm{H}]<-3$ are classified into two subgroups according to their distributions (Yoon et al. 2016). 


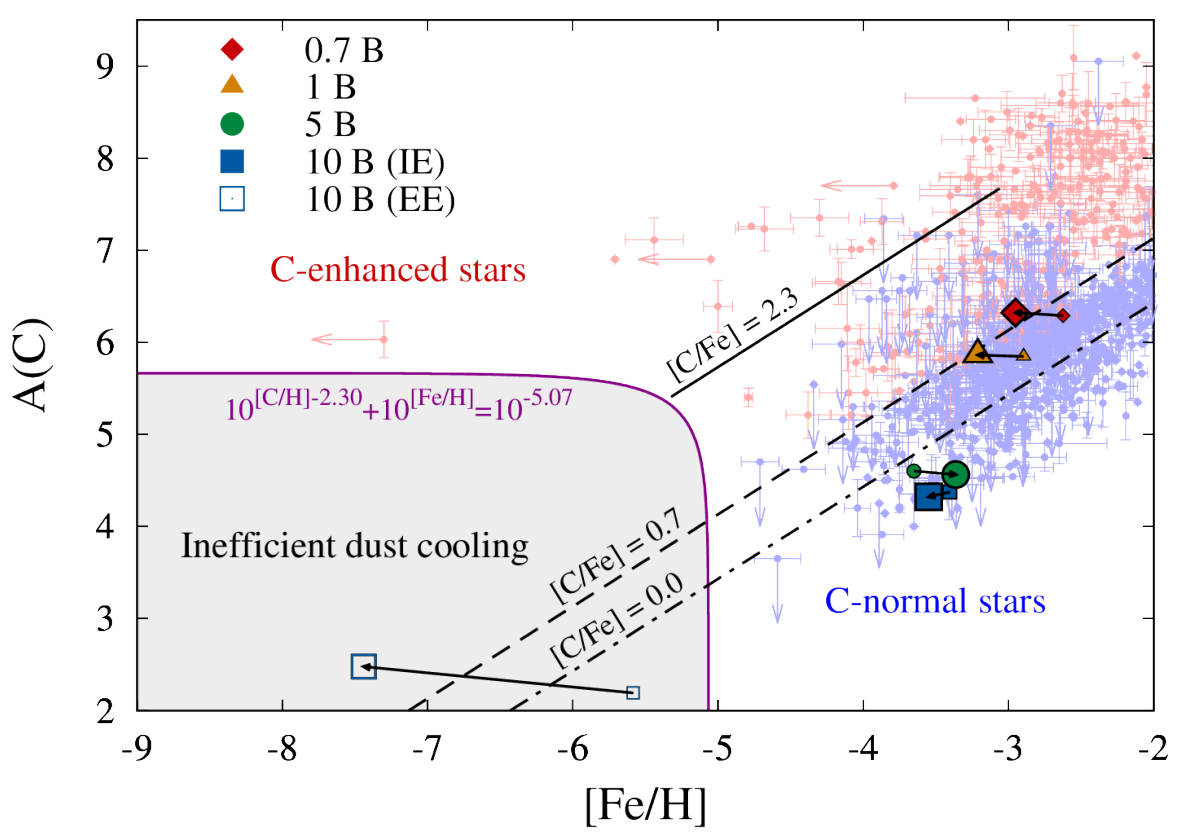

Figure 10. $\mathrm{C}$ and Fe abundances of the enriched clouds from the stratified (large symbols) and uniform (small symbols) ejecta for explosion energies $E_{\mathrm{SN}}=0.7 \mathrm{~B}$ (red), $1 \mathrm{~B}$ (orange), $5 \mathrm{~B}$ (green), and $10 \mathrm{~B}$ (blue). The close and open symbols depict the results in the internally and externally enriched clouds, respectively. The light blue and red dots depict the abundances of observed C-normal and C-enhanced stars, respectively, taken from SAGA database (Suda et al. 2008). The dot-dashed, dashed, and solid lines show the solar abundance $([\mathrm{C} / \mathrm{Fe}]=0)$, the criterion for CEMP stars $([\mathrm{C} / \mathrm{Fe}]=0.7$; Aoki et al. 2007), and the division of Group III and Group II stars $([\mathrm{C} / \mathrm{Fe}]=2.3$; Yoon et al. 2016; Chiaki et al. 2017), respectively. In the grey shaded region below the purple solid curve $10^{[\mathrm{C} / \mathrm{H}]-2.30}+10^{[\mathrm{Fe} / \mathrm{H}]}=10^{-5.07}$, clouds collapse stably against fragmentation for the lack of dust cooling (Chiaki et al. 2017).

Group III stars are distributed around the horizontal line with a constant $A(\mathrm{C})$ while Group II stars are distributed along the line with a constant $[\mathrm{C} / \mathrm{Fe}]$ and just above the region where $\mathrm{C}$-normal stars are distributed. The $A(\mathrm{C})$ show the large star-to-star scatter of $\sigma(A(\mathrm{C})) \sim 1$ dex with a fixed metallicity for all groups. This can be considered to reflect the variation of mass and explosion energy of few progenitor stars in the early stage of galactic chemical evolution (Ryan et al. 1996; Cayrel et al. 2004).

From our simulations, the IE-stars are distributed around the line $[\mathrm{C} / \mathrm{Fe}]=0$ (dot-dashed line) with $\sim 1$ dex deviation. This is consistent with the distribution of observed C-normal and Group II stars. For $E_{\mathrm{SN}} \geq 5 \mathrm{~B}$ (green circle and blue square), the IE-stars have low $[\mathrm{C} / \mathrm{Fe}]$ values (bottom right in Fig. 10) because $[\mathrm{C} / \mathrm{Fe}]$ for a uniform ejecta $(-0.18$ and -0.65$)$ is smaller than the solar abundance ratio, and is unchanged or depleted by the effect of the stratified ejecta. For $E_{\mathrm{SN}} \leq 1 \mathrm{~B}$ (red diamond and orange triangle), $[\mathrm{C} / \mathrm{Fe}]$ is larger $(0.48$ and 0.31$)$ for the uniform ejecta and further enhanced for the stratified ejecta (see Section 3.2.1 ii). The stars are distributed around the critical line for CEMP stars $[\mathrm{C} / \mathrm{Fe}]=0.7$ (dashed line). For $E_{\mathrm{SN}}=0.7 \mathrm{~B}$, the stars would be classified as CEMP stars (red diamond). In this work, we fix the progenitor mass to $25 \mathrm{M}_{\odot}$, but the range of explosion energies $(0.7-10 \mathrm{~B})$ can explain the distribution of observed C-normal and CEMP Group II stars with a scatter of $\sim 1$ dex.

For EE-stars, both $A(\mathrm{C})=2.48$ and $[\mathrm{Fe} / \mathrm{H}]=-7.44$ are smaller than the ones of IE-stars by two orders of magnitude because metals are mixed with a large mass of pristine gas during a merger of three host halo progenitors which induces EE (Section 3.2.2). As a result, $A(\mathrm{C})$ and $[\mathrm{Fe} / \mathrm{H}]$ of the EE-stars exist in the low metallicity region where stars have not been observed (grey shaded region in Fig. 10). We can consider several reasons why these EE-stars with extremely small metallicities have not been observed. First, because the metallicity for IE is larger than that for EE, the C-enhanced abundance pattern resulting from EE may be washed out by the IE of the neighboring cloud itself. Halos only externally enriched may be so rare that EE-stars have not so far been observed among the current samples of EMP stars (Hartwig et al. 2018). Second, clouds with insufficient metal content $(A(\mathrm{C}) \lesssim 6$ ) should contain a negligible amount of dust grains, which is the main coolant that can induce cloud fragmentation. Chiaki et al. (2017) estimate the elemental abundances below which dust cooling works inefficiently as $10^{[\mathrm{C} / \mathrm{H}]-2.30}+10^{[\mathrm{Fe} / \mathrm{H}]}=10^{-5.07}$ (purple curve in Fig. 10). The EE-stars are plotted below this curve, where clouds can collapse stably against fragmentation, and a single massive star $\left(>10 \mathrm{M}_{\odot}\right)$ is likely to form. Such massive stars can not be observed because they will not survive for 13.6 Gyr between the recollapsing redshift $z_{\text {recol }}=22.5$ and the present day.

\subsection{Caveats}

In this work, we perform numerical simulations of the transition from Pop III stars to Pop II stars, considering the non-uniform structure of SN ejecta with realistic nucleosyn- 
thesis models. Because of computational limits, there are several caveats in the simulations.

\subsubsection{Resolution of simulations}

In the simulations, the SPH particle mass is $m_{\mathrm{p}, \text { gas }}=$ $4.45 \mathrm{M}_{\odot}$, and the number of neighboring particles is $N_{\mathrm{ngb}}=$ 64. The number of particles that resolve half of the Jeans length $R_{\mathrm{J}}$ of the collapsing clouds with a density $n_{\mathrm{H}}=$ $10^{3} \mathrm{~cm}^{-3}$ and temperature $T=100 \mathrm{~K}$ is

$\frac{R_{\mathrm{J}}}{h}=3.91\left(\frac{m_{\mathrm{p}, \mathrm{gas}}}{4.45 \mathrm{M}_{\odot}}\right)^{-1 / 3}\left(\frac{n_{\mathrm{H}}}{10^{3} \mathrm{~cm}^{-3}}\right)^{-1 / 6}\left(\frac{T}{100 \mathrm{~K}}\right)^{1 / 2}$

where $h=\left(3 N_{\text {ngb }} m_{\mathrm{p}, \text { gas }} / 4 \pi \rho\right)^{1 / 3}$ is the smoothing length. This resolution is lower than adaptive mesh refinement (AMR) simulations of chemical enrichment (Ritter et al. 2012; Smith et al. 2015; Chiaki \& Wise 2019).

The Strömgren radius is not properly resolved just before the SN explosion (Section 2.2). In an AMR simulation of Ritter et al. (2012), the region within a few pc is fully ionized by a Pop III star with a mass $40 \mathrm{M}_{\odot}$, and the density of the $\mathrm{H}$ II region decreases down to a few $\mathrm{cm}^{-3}$. To resolve the $\mathrm{H}$ II region, we could use refinement techniques, such as particle splitting. Also, de-refinement of the particles are required just before the $\mathrm{SN}$ explosion to follow the expansion of SN shells for the reason stated below. The refinement and de-refinement procedures would induce numerical errors (Chiaki \& Yoshida 2015). Eitherway, at the time of the SN, we inject metals and thermal energy in a sphere with a radius $\sim 3 \mathrm{pc}$ comparable to the expected radius of the $\mathrm{H}$ II region. Therefore, in this work, we do not refine or de-refine particles for the formation of $\mathrm{H}$ II region.

We cannot use particle splitting after a SN explosion to resolve a cooling shell and a recollapsing cloud because particles in the dense regions such as ejecta and shocked shell would be refined and blown away by SN blastwaves to the outer region filled with coarse particles. In the interacting regions between particles with different masses, spurious surface tension would affect the estimation of hydrodynamic force (Saitoh \& Makino 2013). Although we barely resolve the Jeans length of the recollapsing clouds, the elemental abundance ratio for $\mathrm{E} 1$ is consistent with the results of higher-resolution simulations of Ritter et al. (2015) and Sluder et al. (2016) for the same $E_{\mathrm{SN}}$. Also, the lowresolution simulations enable us to investigate the metal enrichment for the wide range of explosion energies $E_{\mathrm{SN}}=0.7-$ $10 \mathrm{~B}$.

\subsubsection{Abundance in inner region of enriched clouds}

Fig. 5 shows that the elemental abundance still has $\sim 1$ dex deviation at half of the Jeans length $R_{\mathrm{J}}$ from the collapse center (black dashed line). Further, $[\mathrm{Fe} / \mathrm{H}]$ systematically decreases or increases toward the center within $R_{\mathrm{J}}$ for E0.7 and E5, respectively. In the succeeding run-away collapse phase, the mass of the cloud core decreases as the maximum density increases (Larson 1969). Therefore, the stars that finally form might have the elemental abundance of the innermost region of the cloud. We estimate the average abundance of the cloud within $R_{\mathrm{J}} / 2$ (red dotted lines). As Table 3 shows, the difference of abundance ratio $\Delta[\mathrm{C} / \mathrm{Fe}]$ becomes larger $\left( \pm 0.6\right.$ dex for IE) than that within $R_{\mathrm{J}}$ (Table 2 ). The region within $R_{\mathrm{J}} / 2$ is resolved only two smoothing lengths of SPH particles (Eq. 7), and thus we can hardly conclude that the abundances would reflect this value. Simulations with higher resolution are thus required to determine the elemental abundances of stars that would eventually form. We will perform AMR simulations to clarify the metal mixing in higher density regions in forthcoming papers.

\subsubsection{Mass and multiplicity of Pop III}

In this paper, we fix the Pop III stellar mass to $25 \mathrm{M}_{\odot}$. This assumption is justified from some numerical simulations showing that the peak of the Pop III mass distribution is around $30 \mathrm{M}_{\odot}$ (Susa et al. 2014; Hirano et al. 2014, 2015). Also, Ishigaki et al. (2018) find that elemental abundances of most EMP stars are best fit with a Pop III hypernova model with a progenitor mass $25 \mathrm{M}_{\odot}$. However, these studies also suggest a range of stellar mass $\left(\sim 10-1000 \mathrm{M}_{\odot}\right)$, which can yield a variety of elemental abundance patterns. We need to study metal enrichment with different Pop III stellar masses to understand the full abundance distribution of EMP stars (Fig. 10). Further, we assume that the MH hosts only a single Pop III star. Recent numerical simulations show that multiple Pop III stars form through the fragmentation of the accretion disk (Turk et al. 2009; Clark et al. 2011; Greif et al. 2012; Susa et al. 2014; Hirano \& Bromm 2017). The elemental abundance of enriched clouds will be the superposition of elemental abundances of multiple progenitor stars (Feng \& Krumholz 2014; Ritter et al. 2015).

We consider a single $\mathrm{MH}$ with a mass $3 \times 10^{5} \mathrm{M}_{\odot}$. Although this is the typical mass of Pop III hosting MHs (Fig. 12 of Chiaki et al. 2018), MHs have a wide mass range from $2 \times 10^{5} \mathrm{M}_{\odot}$ to $3 \times 10^{6} \mathrm{M}_{\odot}$. For low-mass MHs $(\lesssim$ $\left.10^{6} \mathrm{M}_{\odot}\right)$, a massive Pop III star $\left(\gtrsim 100 \mathrm{M}_{\odot}\right)$ can create the $\mathrm{H}$ II region with radii larger than the virial radius, and the SN shell can expand without losing its thermal and kinetic energy. Metals can reach neighboring MHs, and EE mainly occurs. On the other hand, for Pop III stars with masses $\lesssim 100 \mathrm{M}_{\odot}$ and explosion energies $<10 \mathrm{~B}$, IE is the main enrichment channel. For high-mass $\mathrm{MHs}\left(\gtrsim 10^{6} \mathrm{M}_{\odot}\right)$, IE mainly occurs even for massive Pop III stars and energetic SNe (Chiaki et al. 2018).

We do not include the effect of streaming velocity, which in general occurs due to the different evolution of dark matter and baryon density fluctuations before the recombination (Tseliakhovich \& Hirata 2010). This results in the delayed star formation caused by the offset of dense gas clumps from dark matter potential wells (Chiou et al. 2018, 2019; Druschke et al. 2019). Star formation occurs in more massive halos $\left(\sim 10^{7} \mathrm{M}_{\odot}\right)$ than zero-streaming velocity cases, where EE hardly occur even for high $E_{\mathrm{SN}}$ (Chiaki et al. 2018). Previous studies also predict that clouds with surpersonic turbulence may fragment to massive Pop III star clusters (Hirano et al. 2018), indicating that the mixing of metals from multiple sources may be more significant.

\subsubsection{Stars with peculiar elemental abundance}

We use SN models that reproduce the elemental abundances of C-normal EMP stars with $-0.65<[\mathrm{C} / \mathrm{Fe}]_{0}<0.48$. However, $\sim 80 \%$ of EMP stars with metallicities $[\mathrm{Fe} / \mathrm{H}]<-4$ 
Table 3. Metallicity in enriched clouds in inner region

\begin{tabular}{ccccccc}
\hline $\begin{array}{c}{ }^{1} E_{\mathrm{SN}} \\
{[\mathrm{B}]}\end{array}$ & ${ }^{2}$ enrich. & $\begin{array}{c}{ }^{3} R_{\mathrm{J}} / 2 \\
{[\mathrm{pc}]}\end{array}$ & ${ }^{4} A(\mathrm{C})$ & ${ }^{5}[\mathrm{Fe} / \mathrm{H}]$ & ${ }^{6}[\mathrm{C} / \mathrm{Fe}]$ & ${ }^{7} \Delta[\mathrm{C} / \mathrm{Fe}]$ \\
\hline \hline 0.7 & IE & 2.04 & 6.42 & -3.05 & 1.04 & 0.56 \\
1.0 & IE & 2.09 & 5.92 & -3.27 & 0.76 & 0.45 \\
5.0 & IE & 1.95 & 4.64 & -3.05 & -0.74 & -0.56 \\
10.0 & IE & 1.53 & 4.13 & -3.75 & -0.55 & 0.10 \\
& EE & 2.04 & 2.24 & -7.68 & 1.49 & 2.14 \\
\hline
\end{tabular}

Note - (1) Explosion energy. (2) Enrichment mode. (3) Half of the Jeans radius. (4-6) Average carbon and iron abundances and relative abundance ratio $[\mathrm{C} / \mathrm{Fe}]$ within $R_{\mathrm{J}} / 2$. (12) Difference of $[\mathrm{C} / \mathrm{Fe}]$ between the stratified and uniform ejecta.

show C-enhanced abundance pattern (Yoon et al. 2018; Norris \& Yong 2019). Several scenarios have been proposed to explain the CEMP star formation. One is the intrinsic enrichment scenario in which parent clouds of the stars are enriched by C-enhanced interstellar gas. The source of Crich gas is considered to be $\mathrm{SNe}$ with a large fallback of Fe-rich innermost layers into the central remnant and ejection of C-rich outer layers into the ISM (faint SNe; Umeda \& Nomoto 2003). The other is the extrinsic enrichment scenario in which C-rich gas accretes onto the surface of stars from their binary companion in the asymptotic giant branch (AGB) phase (Suda et al. 2004; Komiya et al. 2020).

We can simply apply this work to the faint SN model by assigning the corresponding radial distribution of elemental mass fraction to the metal tracer particles. As Fig. 9 shows, the fraction of metals $f_{\mathrm{enr}}\left(M_{\mathrm{r}}\right)$ incorporated into the enriched ejecta is almost constant in the outer layers but deviated from this constant value in the innermost layers. Since elements lighter than $\mathrm{Fe}$ are dominant in the innermost region of faint SN ejecta, the slight deviation of their abundances might be seen for faint SNe.

Stellar rotation can also modify the elemental abundance in the final phase of stellar evolution. The elemental abundance of materials blown away from the ensuing SN explosion shows C, N, and s-process element enhancement (Meynet et al. 2006; Choplin et al. 2017). In addition, the rotating stars are considered to explode as jet-like $\mathrm{SNe}$. This model is introduced to explain Si-deficient of the star HE $1424-0241$ and Zn-enhancement of the star HE 1327 - 2326 (Tominaga 2009; Ezzeddine et al. 2019). The elemental abundance of clouds enriched from this type of SNe should strongly depend on the direction of the jets against the three-dimensional structure of the intergalactic medium. We will see the enrichment process of jet-like SNe in forthcoming papers.

\section{CONCLUSION}

We perform numerical simulations focusing on the metal enrichment from Pop III SNe, considering a stratified structure of ejecta. Here we consider normal core-collapse supernova $(\mathrm{CCSN})$ models with $-0.65<[\mathrm{C} / \mathrm{Fe}]_{0}<0.48$. We find that SN shells fall back into the central minihalo in all models. The abundance ratio $[\mathrm{M} / \mathrm{Fe}]$ in the recollapsing clouds deviates from that from the uniform ejecta by at most \pm 0.4 dex for any element M. Overall, the fraction $f_{\mathrm{enr}}\left(M_{\mathrm{r}}\right)$ of metals falling back into the recollapsing clouds is almost constant regardless of the initial mass coordinate $M_{\mathrm{r}}$ of the ejecta. The slight deviation from the average abundance of the ejecta is mainly from the turbulent motion of the hot innermost layers. The metallicity range of these clouds is $-3.55<[\mathrm{Fe} / \mathrm{H}]<-2.95$ and resulting $\mathrm{C}$ to $\mathrm{Fe}$ abundance ratio is $-0.56<[\mathrm{C} / \mathrm{Fe}]<0.84$. If the stars directly inherit the elemental abundance of their host clouds, the abundances of the stars are consistent with those of the observed C-normal and Group II CEMP stars.

In addition, for the largest explosion energy (10 B), a neighboring halo is also enriched. Only the outer layers rich with $\mathrm{Ca}$ or lighter elements reach the halo, where the abundance ratio of $\mathrm{C}$ to $\mathrm{Fe}$ is $[\mathrm{C} / \mathrm{Fe}]=1.49$. This means that C-enhanced metal-poor (CEMP) stars can form from the CCSN with the average abundances ratio $[\mathrm{C} / \mathrm{Fe}]_{0}=-0.65$. However, the metallicity of this cloud $[\mathrm{Fe} / \mathrm{H}]=-7.44$ is smaller than in the IE cases by two orders of magnitude because the halo contains a large mass of pristine gas $\left(\sim 10^{5} \mathrm{M}_{\odot}\right)$ before it is enriched. In the low-metallicity region, no low-mass stars have so far been observed. With a statistic analysis, the hypothesis that these stars escape the detection is ruled out at a $99.9 \%$ confidence level with $\sim 500$ samples of EMP stars observed so far (Magg et al. 2019). An explanation of the non-detection is that such a low-metallicity cloud will collapse stably without fragmentation because of a lack of cooling from dust grains. Massive stars are likely to form and not be observed due to their short lifetime compared to the Hubble time (Chiaki et al. 2017).

In this paper, we have studied the metal enrichment from normal CCSNe with a fixed progenitor mass. Nowadays $\sim 10^{6}$ stars have been observed with large survey campaigns, and $\sim 5000$ EMP stars have been identified with follow-up spectroscopic measurements. These observations have revealed that there are various classifications of EMP stars with an enhancement or depletion of specific elements. We will extend our numerical methods to the other types of SN models such as rotating stars, faint SN models and jetlike SN models (Section 4.2.4) to construct the comprehensive formation models of statistical samples of EMP stars. These studies will uncover the general process of chemical enrichment in the ISM and the origins of elements that compose the Universe. 


\section{ACKNOWLEDGMENTS}

We thank John H. Wise for fruitful discussions and comments. GC is supported by Overseas Research Fellowships of the Japan Society for the Promotion of Science (JSPS) for Young Scientists. The numerical simulations and analyses in this work are carried out on XC40 in Yukawa Institute of Theoretical Physics (Kyoto University), Comet in SDSC, Stampede2 in TACC on NSF's XSEDE allocation AST-120046, and PACE/HIVE clusters in the Georgia Institute of Technology. The figures in this paper are constructed with the plotting library GNUPLOT and MATPLOTLIB (Hunter 2007).

\section{DATA AVAILABILITY}

The data underlying this article will be shared on reasonable request to the authors.

\section{REFERENCES}

Abel, T., Bryan, G. L., \& Norman, M. L. 2002, Science, 295, 93 Aoki, W., Beers, T. C., Christlieb, N., et al. 2007, ApJ, 655, 492 Asplund M., 2005, ARA\&A, 43, 481

Asplund, M., Grevesse, N., Sauval, A. J., \& Scott, P. 2009, ARA\&A, 47, 481

Beers, T. C., Preston, G. W., \& Shectman, S. A. 1985, AJ, 90, 2089

Beers, T. C., Preston, G. W., \& Shectman, S. A. 1992, AJ, 103, 1987

Beers, T. C., \& Christlieb, N. 2005, ARA\&A, 43, 531

Belczynski K., Bulik T., Fryer C. L., Ruiter A., Valsecchi F., Vink J. S., Hurley J. R., 2010, ApJ, 714, 1217

Bromm, V., Coppi, P. S., \& Larson, R. B. 1999, ApJ, 527, L5

Caffau, E., Bonifacio, P., François, P., et al. 2011, Nature, 477, 67

Cayrel, R., Depagne, E., Spite, M., et al. 2004, A\&A, 416, 1117

Clark, P. C., Glover, S. C. O., Smith, R. J., et al. 2011, Science, 331,1040

Chen, K.-J., Whalen, D. J., Wollenberg, K. M. J., Glover, S. C. O., \& Klessen, R. S. 2017, ApJ, 844, 111

Chiaki, G., \& Yoshida, N. 2015, MNRAS, 451, 3955

Chiaki, G., Tominaga, N., \& Nozawa, T. 2017, MNRAS, 472, L115

Chiaki, G., Susa, H., \& Hirano, S. 2018, MNRAS, 475, 4378

Chiaki, G., \& Wise, J. H. 2019, MNRAS, 482, 3933

Chiou Y. S., Naoz S., Marinacci F., Vogelsberger M., 2018, MNRAS, 481, 3108

Chiou Y. S., Naoz S., Burkhart B., Marinacci F., Vogelsberger M., 2019, ApJL, 878, L23

Choplin A., Ekström S., Meynet G., Maeder A., Georgy C., Hirschi R., 2017, A\&A, 605, A63

Christlieb, N. 2003, in Reviews in Modern Astronomy, Vol. 16, Reviews in Modern Astronomy, ed. R. E. Schielicke, 191

Cui, X.-Q., Zhao, Y.-H., Chu, Y.-Q., et al. 2012, Research in Astronomy and Astrophysics, 12, 1197

Deng, L.-C., Newberg, H. J., Liu, C., et al. 2012, Research in Astronomy and Astrophysics, 12, 735

Dotani T., et al., 1987, Natur, 330, 230

Douvion T., Lagage P. O., Cesarsky C. J., Dwek E., 2001, A\&A, 373,281

Druschke M., Schauer A. T. P., Glover S. C. O., Klessen R. S., 2019, arXiv, arXiv:1906.09845

Ezzeddine R., et al., 2019, ApJ, 876, 97

Feng Y., Krumholz M. R., 2014, Natur, 513, 523

Frebel A., Norris J. E., 2015, ARA\&A, 53, 631
Gratton R. G., Sneden C., Carretta E., Bragaglia A., 2000, A\&A, 354,169

Greif, T. H., Bromm, V., Clark, P. C., et al. 2012, MNRAS, 424, 399

Hartwig, T., Yoshida, N., Magg, M., et al. 2018, MNRAS, 478, 1795

Hahn, O., \& Abel, T. 2011, MNRAS, 415, 2101

Hirano, S., Hosokawa, T., Yoshida, N., et al. 2014, ApJ, 781, 60

Hirano, S., Hosokawa, T., Yoshida, N., Omukai, K., \& Yorke, H. W. 2015, MNRAS, 448, 568

Hirano, S., \& Bromm, V. 2017, MNRAS, 470, 898

Hirano S., Yoshida N., Sakurai Y., Fujii M. S., 2018, ApJ, 855, 17

Hopkins, P. F. 2013, MNRAS, 428, 2840

Hunter J. D., 2007, CSE, 9, 90

Ishigaki, M. N., Tominaga, N., Kobayashi, C., \& Nomoto, K. 2018, arXiv:1801.07763

Iwamoto K., et al., 1998, Natur, 395, 672

Jappsen, A.-K., Glover, S. C. O., Klessen, R. S., \& Mac Low, M.-M. 2007, ApJ, 660, 1332

Joggerst C. C., Almgren A., Woosley S. E., 2010, ApJ, 723, 353

Keller, S. C., Bessell, M. S., Frebel, A., et al. 2014, Nature, 506, 463

Kitayama, T., Yoshida, N., Susa, H., \& Umemura, M. 2004, ApJ, 613,631

Kitayama, T., \& Yoshida, N. 2005, ApJ, 630, 675

Komiya Y., Suda T., Yamada S., Fujimoto M. Y., 2020, ApJ, 890 , 66

Larson, R. B. 1969, MNRAS, 145, 271

Magg, M., Hartwig, T., Agarwal, B., et al. 2017, arXiv:1706.07054

Magg M., Klessen R. S., Glover S. C. O., Li H., 2019, MNRAS, 487,486

McKee C. F., Ostriker J. P., 1977, ApJ, 218, 148

Meynet G., Ekström S., Maeder A., 2006, A\&A, 447, 623

Norris J. E., Yong D., 2019, ApJ, 879, 37

Omukai, K. 2000, ApJ, 534, 809

Omukai, K., Tsuribe, T., Schneider, R., \& Ferrara, A. 2005, ApJ, 626,627

Ostriker J. P., McKee C. F., 1988, RvMP, 60, 1

Planck Collaboration, Ade, P. A. R., Aghanim, N., et al. 2016, A\&A, 594, A13

Placco V. M., Frebel A., Beers T. C., Stancliffe R. J., 2014, ApJ, 797,21

Ritter, J. S., Safranek-Shrader, C., Gnat, O., Milosavljević, M., \& Bromm, V. 2012, ApJ, 761, 56

Ritter, J. S., Sluder, A., Safranek-Shrader, C., Milosavljević, M., \& Bromm, V. 2015, MNRAS, 451, 1190

Ritter, J. S., Safranek-Shrader, C., Milosavljević, M., \& Bromm, V. 2016, MNRAS, 463, 3354

Ryan, S. G., Norris, J. E., \& Beers, T. C. 1996, ApJ, 471, 254

Saitoh, T. R., \& Makino, J. 2009, ApJ, 697, L99

Saitoh, T. R., \& Makino, J. 2013, ApJ, 768, 44

Schaerer, D. 2002, A\&A, 382, 28

Schneider, R., Ferrara, A., Salvaterra, R., Omukai, K., \& Bromm, V. 2003, Nature, 422, 869

Sluder, A., Ritter, J. S., Safranek-Shrader, C., Milosavljević, M., \& Bromm, V. 2016, MNRAS, 456, 1410

Smith, B., Sigurdsson, S., \& Abel, T. 2008, MNRAS, 385, 1443

Smith, B. D., Wise, J. H., O'Shea, B. W., Norman, M. L., \& Khochfar, S. 2015, MNRAS, 452, 2822

Springel, V. 2005, MNRAS, 364, 1105

Stacy, A., \& Bromm, V. 2014, ApJ, 785, 73

Suda, T., Aikawa, M., Machida, M. N., Fujimoto, M. Y., \& Iben, I., Jr. 2004, ApJ, 611, 476

Suda, T., Katsuta, Y., Yamada, S., et al. 2008, PASJ, 60, 1159

Susa, H. 2006, PASJ, 58, 445

Susa, H., Hasegawa, K., \& Tominaga, N. 2014, ApJ, 792, 32 
Tanaka, S. J., Chiaki, G., Tominaga, N., \& Susa, H. 2017, ApJ, 844,137

Tominaga N., 2009, ApJ, 690, 526

Tominaga, N., Iwamoto, N., \& Nomoto, K. 2014, ApJ, 785, 98

Truelove, J. K., Klein, R. I., McKee, C. F., et al. 1997, ApJ, 489, L179

Tseliakhovich D., Hirata C., 2010, PhRvD, 82, 083520

Turk, M. J., Abel, T., \& O'Shea, B. 2009, Science, 325, 601

Umeda, H., \& Nomoto, K. 2003, Nature, 422, 871

Wadsley J. W., Keller B. W., Quinn T. R., 2017, MNRAS, 471, 2357

Yanny, B., Rockosi, C., Newberg, H. J., et al. 2009, AJ, 137, 4377-4399

Yoon, J., Beers, T. C., Placco, V. M., et al. 2016, ApJ, 833, 20

Yoon, J., Beers, T. C., Dietz, S., et al. 2018, arXiv:1806.04738 